\title{
Management of Pain in Chronic Kidney Disease in Children
}

\author{
Iuliana Magdalena Starcea ${ }^{1,2}$, Anca Viorica Ivanov ${ }^{1 *}$, Maria Adriana Mocanu ${ }^{1}$, Mirabela Sma- \\ randa Alecsa ${ }^{1}$, Teodora Dolhescu ${ }^{2}$ and Ingrith Crenguta Miron ${ }^{1}$ \\ ${ }^{1}$ Department of Mother and Child Medicine, University of Medicine and Pharmacy, Faculty of Medicine, Iasi, Romania \\ ${ }^{2}$ Department of Nephrology and Dialysis, Sfanta Maria Emergency Hospital for Children, Iasi, Romania
}

*Corresponding author: Anca Viorica Ivanov, Department of Mother and Child Medicine, University of Medicine and Pharmacy,

Faculty of Medicine, Iasi, Romania

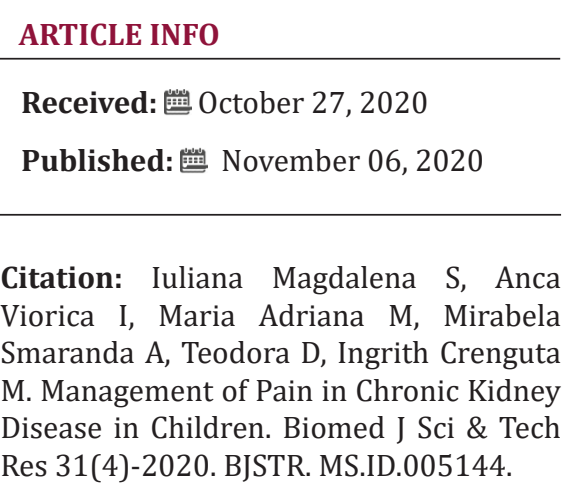

ABSTRACT

Abbreviations: ESRD: End Stage Renal Disease; CKD: Chronic kidney Diseases; WHO: World Health Organization

\section{Introduction}

End stage renal disease (ESRD) is defined as the final stage of many chronic kidney diseases (CKD) and has several course alternatives: death, renal replacement therapy (hemodialysis, peritoneal dialysis), kidney transplantation. Pain is a complex psycho-social phenomenon determined by the interaction of multiple neuroanatomical and neurochemical systems with various cognitive and affective processes. The International Association for the Study of Pain (IASP) defines pain as "an unpleasant sensory and emotional experience associated with actual or potential tissue damage or described in terms of such damage" [1]. Disabling osteodystrophy, calciphylaxis (calcific arteriopathy), development of brown tumor, hypertensive encephalopathy, peripheral neuropathy, generalized edema, are only few complications associated to end stage renal disease with strong debilitating potential. Another common problem of patient with CKD is related to invasive investigations and treatments. The patient repeats the painful experience whenever he returns for investigation or renal replacement therapy, which may increase his anxiety and repulsion. For this reason, is important that all measures be taken for a psychological and analgesic preparation from the first experience, in order that side effects should be anticipated by the patient as minimal (Doctor-patient relationship, Iorga [2]). For all these reasons pain is very common in CKD and ESRD patients; most of these subjects are still under diagnosed and undertreated.

The perception of pain, along with somatic changes induced by CKD, shapes the character of the chronic kidney disease child. In this sense, medical therapy of the chronic kidney disease must be backed up by adequate psychological support. Proper pain management is mandatory for a better quality of life of this patient. Quality of life can be understood according to the WHO definition of health, in the sense of physical, mental and social well-being, and there are situations when a good QoL can be achieved even if the patient has a chronic condition.. Improving the quality of life is the goal of chronic dialysis patient care and the measure of its quality. The child's perception of quality of life must be understood and respected, it depends primarily on the basic processes of cognitive development.

We conducted a small retrospective, nonrandomized study about impact of chronic pain on the quality of life of the chronic 
dialysis patient. The study focused on the pediatric patients with ESRD monitored in the Nephrology Department of the St. Mary's Emergency Clinical Hospital for Children, Iasi, a representative unit for the Region of Moldova, Romania. The study included 28 patients with ESRD, aged between 0 and 18 years, followed for a period of 5 years, 2015 - 2019. 28\% were treated by peritoneal dialysis, and $72 \%$ by hemodialysis, of which $60 \%$ on fistula arteriovenous, the rest on the long-life central catheter. The Pediatric Quality of Life Inventory and Illness Perception Questionnaire was applied to assess quality of life and perception of the disease. The available multidimensional questionnaires (Brief Pain Inventory, McGill Pain Questionaire, Wong - Baker facial assessment scales, Oucher scales) [3] allow the assessment of pain by assessing the intensity, frequency, duration, quality and sensitivity of the child's pain. Pain overcoming strategies as well as the impact of pain on the patient's daily activity are also estimated. The pain was present in the evolution of dialysis patients, being related to the pathology of the venous approach and to infectious or thrombotic complications of the central venous catheterization. One of the patients developed complications related to the Gore Tex graft placed as a venous approach for hemodialysis. Complications associated with AV grafts included local hematoma, but also lower limb edema and neuropathy, all accompanied by significant pain. The therapy was a combination of anti-inflammatory drugs, carbamazepine and gabapentin, but unsatisfactory control required intermittent opioid use. In 4 patients the recurrent development of secondary peritonitis required change of dialysis method, then access to a kidney transplant. Sever pain needed in this case opioid use, along with antibiotic therapy. Sever renal osteodystrophy complicated the evolution in 4 cases of hemodialysis patients. Several patients in the chronic extrarenal clearance program for more than 5 years have developed secondary neuropathy, requiring opioid and gabapentin control, as well as associated antidepressant medication. One of the patients developed septic necrosis of the femoral head, requiring prosthesis, and another suffered multiple fractures after a minor injury from falling. In the same patient, calciphylaxis and adenomatous development of the parathyroid glands were highlighted, requiring 2-stage surgical resection and subsequent bisphosphonate therapy, after monitoring the case together with the Endocrinology Clinic. Another two patients died with severe calciphylaxis, uncontrolled by the therapy. They complained of intense pain due to respiratory restriction by pulmonary metastatic calcifications, but also by phenomena of vascular ischemia secondary to mediocracies and increased arterial stiffness, especially in the coronary and cerebral territory.
A recent review (Pain Management, [4]) highlights pain medication that are safe, but in adjusted dose, as well as drugs to be avoided in the management of pediatric kidney disease. Modifications in the prescription of some analgesics are required in CKD children, due to problems associated with reduced drug or metabolite elimination [5]. Acetaminophen should be used as a first-line therapy for pain management in children with CKD. In dialyzed patients, these opioids should be considered as secondline agents and patients should be carefully monitored. Opioid induced constipation can be managed with peripherally-acting-nopioid-receptor-antagonists. (Safe Use of Opioids in Chronic Kidney Disease and Hemodialysis Patients: Tips and Tricks for Non-Pain Specialists, By: Coluzzi Flaminia [6]). Opioids may be added to control moderate to severe pain. Because the nephrologist is a non a pain specialist, may be unfamiliar with different chronic pain syndromes and with safe and appropriate use of opioids. It should consider referral to pain specialists when pain is not adequately managed with standard analgesics. A multidisciplinary team should be involved in the management of vulnerable patients suffering from CKD or ESRD. Unfortunately, the number of clinical trials available on the use of opioids in ESRD in children is still limited, and extrapolating information by clinical studies on different children populations may not be prudent. Therefore, further trials are warranted to evaluate the efficacy and safety of opioids in CKD and ESRD patients.

\section{References}

1. (2019) IASP's Proposed New Definition of Pain Released for Comment.

2. Magdalena Starcea, Magdalena Iorga, Laszlo-Zoltan Sztankovszky, Mihaela Munteanu (2014) The doctor - patient relationship in childrens chronic kidney disease and itd importance for the quality of life for the dialysis patient, European Journal of Science and Theology 10(3): 27-36.

3. Magdalena Iorga, Magdalena Starcea, Mihaela Munteanu, Laszlo-Zoltan Sztankovszky (2014) Psycological and social problems of children with chronic kidney disease, European Journal of Science and Theology 10(1): 179-188.

4. Amanda Reis, Caitlyn Luecke, Thomas Keefe Davis, Aadil Kakajiwala (2018) Pain Management in Pediatric Chronic Kidney Disease, J Pediatr Pharmacol Ther 23(3): 192-202.

5. Phuong-Chi T Pham, Edgar Toscano, Phuong-Mai T Pham, et al. (2009) Pain management in patients with chronic kidney disease, NDT Plus 2: 111-118.

6. Flaminia Coluzzi, Francesca Felicia Caputi, Domenico Billeci (2020) Safe Use of Opioids in Chronic Kidney Disease and Hemodialysis Patients: Tips and Tricks for Non-Pain Specialists, Therapeutics and Clinical Risk Management 16: 821-837. 
ISSN: 2574-1241

DOI: 10.26717/BJSTR.2020.31.005144

Christos P Beretas. Biomed J Sci \& Tech Res

(C) This work is licensed under Creative By Commons Attribution 4.0 License

Submission Link: https://biomedres.us/submit-manuscript.php

$\begin{array}{ll}\text { BIOMEDICAL } & \text { Assets of Publishing with us } \\ \text { RESEARCHES } & \text { - Global archiving of articles } \\ \text { - Immediate, unrestricted online access } & \text { - Rigorous Peer Review Process } \\ & \text { - Authors Retain Copyrights } \\ \end{array}$

